\title{
Habitus en torno a la evaluación. (Des)encuentro entre medición y comprensión
}

\author{
Institutional Habitus Around the Evaluation. (Des)Encounter Between Measurement \\ and Understanding
}

Hábitus institucionais relativos à avaliação. (Des)encontro entre medição e compreensão

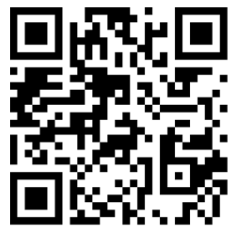

Gerardo Ignacio Sánchez-Sánchez

Universidad Católica del Maule

Talca, Chile

gerignsan@gmail.com

https://orcid.org/0000-0003-1694-1406

Ximena Elizabeth Jara-Amigo Universidad Autónoma de Chile

Talca, Chile

xjaraa@uautonoma.cl

https://orcid.org/0000-0001-7695-7579

Recibido • Received • Recebido: 31 / 01 / 2020

Corregido • Revised • Revisado: 14 / 06 / 2021

Aceptado • Accepted • Aprovado: 05 / 07 / 2021

\begin{abstract}
Resumen:
Objetivo. El objetivo es comprender el habitus que las organizaciones escolares desarrollan en torno a la evaluación educativa. El trabajo se realizó con docentes de educación especial, preescolar, primaria y secundaria. Las propias percepciones y concepciones teóricas y prácticas del profesorado sobre los diversos ámbitos de su quehacer pedagógico están definidas por la institución donde trabaja y las influencias que esta genera. Metodología. La investigación se desarrolló con los parámetros de la metodología cualitativa, de tipo transeccional con un nivel de profundidad exploratoria-descriptiva. Mediante una aproximación de carácter intensivo, se utilizó la entrevista semiestructurada a 40 profesores en programas de postgrado, buscando el significado de las experiencias del personal docente. La información se procesó a través del análisis narrativo. Discusión. Los resultados muestran la presencia de un habitus paradojal en torno a la evaluación, que la configura como cultura de medición, cuantificación y control, lo cual resta oportunidades para transformarla en espacio de debate, reflexión y colaboración. Conclusiones. Se concluye que la evaluación desarrollada en los contextos organizacionales de la escuela se debate entre el (des)encuentro de sus funciones sociales y sus funciones pedagógicas. La proyección de resultados muestra que la evaluación constituye, en los procesos de formación inicial y continua del profesorado, uno de los talones de Aquiles de la práctica educativa. En el ámbito de las intenciones, se perfila la necesidad urgente de convertirla en un proceso que posibilite la función pedagógica, que integre las unidades de enseñanza, aprendizaje y evaluación.
\end{abstract}

Palabras claves: Evaluación educativa; habitus institucional; profesorado; institución educativa; Chile. 
http://doi.org/10.15359/ree.26-1.2

http://www.una.ac.cr/educare

educare@una.ac.cr

\begin{abstract}
:
Objective. The goal is to understand the habitus that school organizations develop around educational evaluation. The work was done with special education, preschool, primary, and secondary teachers. The teachers' own theoretical and practical perceptions and conceptions of the various areas of their pedagogical work are defined by the institution where they work and its influences. Method. The research was developed with qualitative methodology parameters of transeccional type with a level of exploratory-descriptive depth. Through an intensive approach, the semi-structured interview was applied to 40 teachers in postgraduate programs, seeking the meaning of the teaching staff's experiences. The information was processed through narrative analysis. Discussion. The results show the presence of a paradoxical habitus around evaluation, which configures it as a culture of measurement, quantification, and control, subtracting opportunities to transform it into a space of debate, reflection, and collaboration. Conclusions. It is concluded that the evaluation developed in the organizational contexts of the school is debated between the (dis)agreement of its social functions and its pedagogical functions. The projection of results shows that the evaluation constitutes, in teachers'initial and continuous training processes, one of the weaknesses of the educational practice. In the field of intentions, the urgent need to turn it into a process that enables pedagogical function is outlined, integrating the teaching, learning, and evaluation units.
\end{abstract}

Keywords: Educational evaluation; institutional habitus; professorate; educational institution; Chile.

\title{
Resumo:
}

Objetivo. O objetivo é entender o habitus que as organizações escolares desenvolvem em torno da avaliação educacional. $O$ trabalho foi realizado com professores de educação especial, préescola, ensino fundamental e médio. As próprias percepções e concepções teóricas e práticas dos professores dos vários campos de seu trabalho pedagógico são definidas pela instituição em que trabalham e pelas influências que ele gera. Metodologia. A pesquisa foi desenvolvida com os parâmetros da metodologia qualitativa, do tipo transacional, com nível de profundidade exploratório-descritivo. Utilizando uma abordagem intensiva, foi utilizada a entrevista semiestruturada de 40 professores em programas de pós-graduação, buscando o significado das experiências dos docentes. As informações foram processadas por meio de análise narrativa. Discussão. Os resultados mostram a presença de um hábitu paradoxal em torno da avaliação, que o configura como uma cultura de medição, quantificação e controle, subtraindo oportunidades para transformá-lo em um espaço de debate, reflexão e colaboração. Conclusões. Conclui-se que a avaliação desenvolvida nos contextos organizacionais da escola é debatida entre o (des) encontro de suas funções sociais e suas funções pedagógicas. A projeção dos resultados mostra que a avaliação constitui, nos processos de formação inicial e contínua de professores, um dos calcanhares de Aquiles da prática educacional. No campo das intenções, destaca-se a necessidade urgente de transformá-lo em um processo que possibilite a função pedagógica, integrando as unidades de ensino, aprendizagem e avaliação.

Palavras-chave: Avaliação educacional, habitus institucional, corpo docente, instituições educativa, Chile. 
http://doi.org/10.15359/ree.26-1.2

\section{Introducción y marco teórico}

Los centros educativos constituyen campos de experiencias, decisiones y prácticas que condicionan los procesos que, en su interior, tienen lugar; razón por la cual las disposiciones individuales desarrolladas por el profesorado están mediadas, necesariamente, por las prácticas organizativas, vale decir, "el profesorado no actúa solo ni de forma independiente a su contexto de referencia; al contrario, está imbricado en contextos institucionales" (Tarabini et al., 2015, p. 39).

Este contexto configura lo que Bourdieu (1998) define como habitus. Expresión clave en tanto articula lo individual y lo social, vale decir, las estructuras internas asociadas a la subjetividad y la estructura social externa. A partir de este concepto, Reay et al. (2001) proponen el de habitus institucional para referir al conjunto de predisposiciones, esquemas y expectativas que configuran las instituciones educacionales, y a partir del cual es posible lograr una comprensión más compleja y situada de la institución escolar y de sus procesos constitutivos. La burocracia, por una parte, y la lógica disciplinaria, por otra, configuran los aspectos centrales en las organizaciones escolares y los habitus que definen.

Esta organización aparece fuertemente tensionada en un escenario de postmodernidad, pues son otras las condiciones que presiden el desarrollo de las sociedades contemporáneas. En ese sentido, las instituciones responsables de la socialización de la infancia"ahora desaparecen, se repliegan, o simplemente se inhiben y pierden eficacia a este respecto, lo que hace que aumenten las funciones y la carga de trabajo que ha de soportar la escuela" (Vera Vila, 2007, p. 13). Las instituciones enfrentan el desafío de recuperar su capacidad de funcionar como marcos de referencia que regulan y protegen las acciones, las decisiones, las relaciones y las prácticas. Sin embargo, la tarea no resulta del todo fácil, pues sus habitus las tienden a anclar a prácticas reactivas derivado de lo cual, "la escuela ... se ha convertido en una institución sobredemandada y subdotada" (Tenti Fanfani, 2004, p. 40).

Con serias dificultades para responder a los requerimientos sociales actuales, la institución educativa tiende a la continuidad de su estructura (Alliaud, 2017).

Esla permanencia casi intacta de esta estructura moderna lo que explica su disfuncionalidad en el presente y exacerba las contradicciones que llevó inscriptas desde sus orígenes; perpetúa, de esa manera, ciertos habitus en relación con las gramáticas y procesos desarrollados. El habitus obliga a entender el fuerte condicionamiento ejercido desde la cultura escolar; cultura que, desde la perspectiva de Pérez Gómez (2005), se define como "el conjunto de creencias, valores, hábitos y normas dominantes que determinan lo que [un] grupo social considera valioso en su contexto profesional, así como los modos políticamente correctos de pensar, sentir, actuar y relacionarse entre sí" (p. 162). 
http://doi.org/10.15359/ree.26-1.2

http://www.una.ac.cr/educare

educare@una.ac.cr

Las escuelas son creadoras de habitus, generadoras de cultura y, por tanto, tienden a condicionar (posibilitar o limitar) los diversos procesos que en su interior tienen lugar. En el caso chileno y como producto de las políticas educacionales impulsadas, se ha configurado una cultura escolar y un rol docente en que la calidad educativa "pasó a ser colonizada por la lógica de un currículum nacional y de estándares (y mediciones) de aprendizaje" (Assaél Budnik et al., 2011, p. 315). De esa manera, "incorpora exámenes nacionales apoyados en motivaciones extrínsecas, desarrollándose un trabajo escolar orientado a responder tales pruebas, derivando en estrechamiento del currículum, individualizando y desprofesionalizando la docencia" (Ruffinelli Vargas et al., 2017, p. 23).

\section{El habitus construido en torno a la evaluación}

Uno de los procesos distintivos de la institución escolar y que contribuye al desarrollo de su racionalidad lo constituye la evaluación, la cual no escapa al habitus institucional y, en definitiva, a las brechas entre lo que declara y pretende, y lo que en definitiva termina siendo en su gramática y en su práctica.

En la educación formal, la práctica de la evaluación está centrada en dilucidar el saber del estudiantado, y no necesariamente en acompañar su aprendizaje. Su práctica indica:

Comúnmente se toman pruebas en momentos específicos. Pruebas que preguntan todo tipo de cosas y que los(as) estudiantes deben responder, en función de patrones de respuestas que se consideran correctas o apropiadas y que el(la) profesor(a) utiliza como parámetro de comparación para asignar valor a sus respuestas. Esta valoración suele traducirse en una calificación, que forma parte de un sistema de calificaciones que determina premios, castigos, reconocimientos de diversa naturaleza y la promoción de los(as) estudiantes, en diferentes cursos y niveles. Finalmente, el historial y trayectoria del(la) estudiante en este sistema, determina su titulación que, en el fondo es una credencial para acceder a un "mercado" de nuevas oportunidades académicas y de condiciones laborales. (Rioseco Pais, 2016, pp. 81-82)

Seconfigura, así, un habitus de la evaluación centrado en clasificary cosificaral estudiantado; desconoce, con ello, que el aprendizaje tiene lugar en un sujeto en situación, con una historia que condiciona aquello que es y que sabe; esto, desde la perspectiva de Tardif (2004), supone reconocer la existencia de un trabajo docente constituido por dilemas y tensiones.

De esa manera, la evaluación que se administra en muchos de los sistemas educativos "deshumaniza la educación, ya que mide y compara a los sujetos, con el objetivo principal de clasificarlos para condicionar sus oportunidades en el futuro" (Rioseco Pais, 2016, p. 84). En consecuencia, se configuran determinadas representaciones del sujeto que participa del proceso 
http://doi.org/10.15359/ree.26-1.2

formativo. Específicamente se observan limitaciones para entender que, aunque se enseñe a grupos, el profesorado no puede dejar de tener en cuenta las diferencias individuales, pues son las personas las que aprenden; que son heterogéneas, y que no poseen las mismas capacidades personales ni las mismas posibilidades sociales; y que también el estudiantado son seres sociales cuyas características socioculturales despiertan actitudes y juicios de valor en el personal docente, y que, en definitiva, todas estas representaciones configuran prácticas y habitus que median los procesos de enseñanza, de aprendizaje y, fundamentalmente, de evaluación.

Más allá de los cuestionamientos a la práctica, lo cierto es que la evaluación constituye un componente central de un proceso de enseñanza-aprendizaje de calidad. Su concepto en el campo de la educación ha ido evolucionando producto de la incorporación de nuevas comprensiones y variables asociadas a contextos y procesos.

Desde la perspectiva de Förster (2017), la concepción de evaluación muestra una evolución que puede ser clasificada en tres momentos temporales. Antes de los años noventa prevalece una noción de evaluación regida por las teorías y práctica de la medición educacional y el fuerte influjo de las teorías de corte conductista, por tanto, el énfasis de la evaluación estaba en la selección y el control y, por ende, en su rol sumativo. Entre la década del 90 e inicios del año 2000, la evaluación recibe la influencia del sistema relacionado con la rendición de cuentas y la estandarización. Desde el 2000 a la fecha se alude a evaluación del aprendizaje y para el aprendizaje; se releva la función formativa y la retroalimentación efectiva. Ello resulta coincidente con el planteamiento de Esquivel (2009), quien reconoce un primer momento en que la evaluación está disociada de la enseñanza y el aprendizaje, aplicada al final de un curso con la intención de comprobar los logros de aprendizajes fundamentados en la tradición psicométrica, adoptada por la psicología educativa; un segundo momento, en que el maestro o maestra se convierte en protagonista en la rendición de cuentas y, con ello, una evaluación fundamentada en sistemas nacionales de evaluación del logro del estudiantado. Y en la actualidad, la noción de evaluación para el aprendizaje y, por tanto, al servicio de decisiones pedagógicas con la intención de regular en forma continua el aprendizaje.

Este avance en las concepciones sobre evaluación debe finalmente expresarse en las prácticas evaluativas que se desarrollan en los contextos escolares, en los cuales es posible constatar la presencia de una evaluación que puede servir a distintos fines y generar diversos efectos en los procesos de enseñanza-aprendizaje.

Existe la posibilidad de utilizar la evaluación para diversas y muy diferentes funciones: Diagnóstico, comparación, clasificación, jerarquización, control, mejora, comprensión, amenaza, comprobación, aprendizaje, emulación, diálogo, clasificación, pasatiempo, etc. Salta a la vista que no todas tienen la misma naturaleza e importancia. Unas son abiertamente desechables; otras, sin duda deseables. (Santos Guerra, 1999, p. 40) 
http://doi.org/10.15359/ree.26-1.2

http://www.una.ac.cr/educare

educare@una.ac.cr

Dentro de estas posibilidades, es posible reconocer que la evaluación se mueve entre dos lógicas: las que, desde la perspectiva de Perrenoud (2008), pueden significar la presencia de una evaluación al servicio de la selección, según lo cual "los alumnos se comparan, y luego se clasifican, en virtud de una norma de excelencia" (p. 10); o al servicio de los aprendizajes y, por tanto, "instrumento privilegiado para una regulación continua de las intervenciones y las situaciones didácticas" (Perrenoud, 2008, p. 14); esto es, una evaluación capaz de reconocer y valorar la diversidad presente en los contextos escolares.

En correspondencia con los planteamientos actuales sobre aprendizaje y enseñanza, favorecer una práctica evaluativa vinculada a la posibilidad cierta de regular el aprendizaje en términos formativos supone reconceptualizarla como un camino para el aprendizaje. Es decir, un proceso que favorece la toma de decisiones de naturaleza pedagógica y didáctica (Esquivel, 2009).

Coincidente con ello, Chaviano Herrera et al. (2016) confirman que

la tendencia actual es la de concebir la evaluación desde una perspectiva comprehensiva en cuanto a su objeto, funciones, metodología y técnicas, participantes, condiciones, resultados, efectos y determinantes. Se manifiesta con fuerza el reconocimiento de su importancia social y personal desde un punto de vista educativo, formativo, así como para el propio proceso enseñanza aprendizaje por el impacto que tiene el modo de realizarla y la forma en que el estudiante la percibe. (p. 195)

La evaluación constituye un componente central del buen aprendizaje en la medida que se asocia a retroalimentación, formación y regulación (Hargreaves et al., 2000). Avanzar en esa dirección implica gestionar adecuadamente las funciones de la evaluación:

Por una parte, la función social de certificación y por otra, la función pedagógica, de regulación continua del aprendizaje. Funciones que en general, se incrementan en cantidad con la misma fuerza que van perdiendo en transparencia, condicionando la actividad de enseñanza y de aprendizaje. (Sánchez-Sánchez y Jara-Amigo, 2019, p. 66)

Frente a ello, el profesorado desarrolla los procesos de enseñanza, aprendizaje y evaluación enfrentando los requerimientos y condicionamientos de un habitus institucional que lo condiciona:

El sistema escolar está constantemente pidiendo al docente que gestione los resultados de sus evaluaciones, y que tome decisiones respecto de la efectividad de las prácticas de enseñanza desde las evidencias de aprendizaje de sus estudiantes, pero el análisis siempre se realiza a partir de las calificaciones obtenidas y no desde el avance o logro en los aprendizajes esperados. (Förster, 2017, p. 11) 
http://doi.org/10.15359/ree.26-1.2

El desafío que enfrenta la evaluación se explica dada la existencia de una cultura de la evaluación que la mantiene ancorada a un tipo de racionalidad que no logra responder a los cambios sociales que "han disparado las demandas educativas sobre la escuela, han cambiado las funciones de los profesores y han puesto de manifiesto la insuficiencia de la cultura escolar de la modernidad" (Esteve, 2003; Esteve et al, 1995; Vera y Esteve, 2001, citados en Vera Vila, 2007, p. 11).

En ese contexto, esta investigación sobre evaluación del aprendizaje con el profesorado de educación especial, preescolar, primaria y secundaria se orienta a la indagación del habitus que rodea a la evaluación en términos de los des(encuentros) entre la cultura de la evaluación como medición y comprensión. En esa línea, comprender las tensiones y posibilidades que presenta la práctica evaluativa para responder a los desafíos de diversidad, inclusión y retroalimentación en los procesos de formación inicial y continua de profesorado considerando que se trataría de una práctica que condiciona el funcionamiento del aula y las decisiones que se toman en relación con los procesos de aprendizaje y enseñanza.

\section{Metodología}

Este artículo informa parte de la investigación "los docentes en procesos de formación de postgrado y la reflexión sobre el quehacer en las escuelas" (2017-2018), desarrollada en el contexto de una Facultad de Ciencias de la Educación. El análisis cualitativo empleó el microanálisis de datos propuesto por Corbin y Strauss (2008). Se trabajó con 40 docentes en ejercicio, quienes ofrecieron sus representaciones respecto a la evaluación en el contexto del sistema educacional chileno. De este grupo, 26 corresponden a mujeres y 14 varones, con edades que oscilan entre los 25 y 45 años. Los criterios para elegirlos fueron estar cursando un programa de maestría en el ámbito de la educación, con experiencia en el sistema escolar y que aceptaran participar de forma voluntaria, previa firma de consentimiento. Desde el punto de vista de la vinculación con la docencia, el 37,5\% (15 docentes) lleva menos de cinco años de experiencia; el 37,5\% entre 6 y 10 años de experiencia; y el 25\% declara tener más de 10 años de experiencia en el sistema escolar. La totalidad de docentes declara trabajar en jornada completa de 44 horas semanales. Respecto al nivel educativo en que se ejerce la docencia, el 12,5\% (5) son educadoras de párvulos; el 15\% (6) son docentes diferenciales; el 37,5\% (15) corresponde a educación básica; y el 35\% (14) a educación media.

Se optó por una aproximación cualitativa partiendo del "supuesto básico de que el mundo social es un mundo construido con significados y símbolos, lo que implica la búsqueda de esta construcción y de sus significados" (Ruiz Olabuénaga 2009, p. 31). El abordaje metodológico requirió una aproximación de carácter intensivo mediante el uso de entrevista semiestructurada (Flick, 2007) a profesionales de la educación, que indagó en torno a la intencionalidad de la evaluación y, particularmente, la cultura que termina por instalar, así como también se explora en las oportunidades de desarrollo que presenta. 
http://doi.org/10.15359/ree.26-1.2

http://www.una.ac.cr/educare

educare@una.ac.cr

El análisis de las entrevistas fue realizado a partir de la teoría fundamentada. Administradas las entrevistas, se procedió a: su transcripción, categorización de la información, sistematización, análisis y reducción de los datos los que, de acuerdo con los planteamientos de Corbin y Strauss (2008), fueron trabajados al amparo de una aproximación de naturaleza inductiva a la realidad, que facilitó la identificación de categorías adecuadas a la investigación para el correspondiente análisis de contenido.

\section{Resultados}

Las categorías de análisis que surgen de las entrevistas a los sujetos (Tabla 1) dan origen a los siguientes resultados.

Tabla 1: Tópicos y categorías en torno a la cultura evaluativa

\begin{tabular}{|c|c|c|}
\hline & Tópico & Categorías \\
\hline & Medición & 1.1 Individualismo \\
\hline & & 1.2 Competitividad \\
\hline & & 1.3 Cuantificación \\
\hline & & 1.4 Simplificación \\
\hline & Comprensión & 2.1 Autocrítica \\
\hline & & 2.2 Debate \\
\hline & & 2.3 Flexibilidad \\
\hline & & 2.4 Colegialidad \\
\hline
\end{tabular}

Nota: Elaboración propia, tomando en cuenta entrevistas aplicadas.

\section{Cultura de la evaluación como medición}

Esta cultura de la evaluación entendida como medición es definida en las categorías de individualismo, competitividad, cuantificación y simplificación.

\section{Individualismo}

El individualismo aparece en los relatos como componente de la cultura de la evaluación. Se lo considera condicionante a la hora de pensar en procesos de evaluación que sirvan a la toma de decisiones y generen impactos en la mejora del aprendizaje del estudiantado.

Desde la perspectiva de las personas entrevistadas, el componente individualista lo experimentan ambos actores, vale decir, el profesorado que resuelve la evaluación en solitario, y el estudiantado que la vive como un proceso personal. De acuerdo con los relatos: 
http://doi.org/10.15359/ree.26-1.2

... La cultura de la evaluación es individualista, desde dos frentes, por un lado, el docente afronta la difícil misión de evaluar en solitario, no comparte, ni comenta los resultados con sus pares, y por otro, el alumno es evaluado individualmente, hasta cuando realiza trabajos grupales, en definitiva, la hora de la verdad, se afronta sin la ayuda de los compañeros y sin la cooperación de la clase. (S16)

En cuanto a las implicancias que esta dimensión de la cultura evaluativa muestra, emergen con fuerza entre los sujetos entrevistados, la falta de colaboración y la imposibilidad de cambio. Más específicamente:

... Esta tendencia al individualismo condiciona y limita la posibilidad de cambio, ya que no permite la generación de intercambios o trabajo colaborativo, centrándose solo en esfuerzos particulares. Por tanto, mientras este sea el enfoque no se podrá avanzar en la educación. (S20)

... El individualismo que se observa en torno a la evaluación, inhibe la cooperación, fomenta la competencia, impide la complementariedad. Además, limita la reflexión, y con ello, las posibilidades de mejora. (S23)

\section{Competitividad}

Asociada al individualismo y como elemento concomitante, se instala la competitividad que tiende a impregnar los procesos formativos de determinados énfasis y efectos vinculantes.

En cuanto a los énfasis que termina por adoptar el proceso de enseñanza - aprendizaje figuran los conceptos y prácticas asociadas a control, selección e incluso jerarquización, las cuales resultan altamente coincidentes con las características que, en la actualidad, presentan los sistemas sociales en su conjunto. Según los participantes:

... No permite que la evaluación sea entendida como un proceso colectivo reflexivo, abierto al error como elemento de aprendizaje. Al contrario, la obtención de un producto sin importar los medios por los cuales se obtiene se vuelve relevante puesto que esos resultados me situarán en una posición de ventaja o desventaja respecto a un entorno cuyo único objetivo es sobrepasar mi posición, lo que me lleva en forma muchas veces inconsciente a pensar en el resto como adversario y no como el otro con quien yo puedo construir y aprender. (S19)

Esta competencia que termina por configurar un rasgo distintivo del habitus institucional, la experimenta en el aula el estudiantado, en la escuela el profesorado y en la sociedad, la propia institución. Desde los planteamientos de los participantes: 
http://doi.org/10.15359/ree.26-1.2

http://www.una.ac.cr/educare

educare@una.ac.cr

... Prevalece el concepto de competitividad tanto para los alumnos (entre pares), como para los establecimientos (medición a través de pruebas estandarizadas), lamentablemente no considerando la evaluación como centro en el aprendizaje, sino instrumentalmente como un canje. (S21)

En consecuencia, se desencadenan prácticas evaluativas que reflejan los propios condicionamientos sociales. Así lo entienden y manifiestan los sujetos entrevistados:

... Al revisar la cultura del sistema de educación en Chile, podemos observar, que éste a su vez, es una réplica de lo que es la sociedad actual, donde predomina la competitividad. Esto ha hecho a su vez que exista individualismo. Estas conductas se perpetúan y actualmente, parece imposible revertir esta situación, en la que las personas se ven inmersas en una sociedad exitista que valora esa competitividad. (S3)

... La competitividad se ha instalado en nuestra sociedad, esto se desarrolla a través de las evaluaciones estandarizadas, como lo son el SIMCE, INICIA, PSU. Con dichas evaluaciones se termina comparando mucho y logrando muy poco cambio. (S14)

\section{Cuantificación}

La cultura de cuantificación viene a complementar las características anteriores y se revela en la tendencia a medir y expresar en números el aprendizaje logrado por el estudiantado, con el correspondiente reduccionismo de un proceso complejo como es el aprendizaje.

En este sentido, el énfasis de la evaluación se instala preferentemente en los resultados y, con ello, se tienden a desestimar las dimensiones potenciales del proceso. Así lo manifiestan los sujetos entrevistados:

... En nuestra cultura evaluativa todo se reduce a cuantificación, y a evaluaciones centradas solo en resultados, lo que dificulta y termina por impedir la reflexión de los profesores y del sistema en su conjunto; en consecuencia, no hay un proceso que permita dimensionar verdaderamente lo que están aprendiendo los estudiantes. (S27)

... La práctica de la cuantificación condiciona las posibilidades de cambio porque el centro de la evaluación de los aprendizajes tiene como foco la calificación, y no las necesidades diversas de los estudiantes. (S7)

Esta cuantificación se ve reflejada, no solo a nivel de aula, sino fundamentalmente a nivel de sistema educativo, como lo evidencian los siguientes relatos: 
http://doi.org/10.15359/ree.26-1.2 http://www.una.ac.cr/educare educare@una.ac.cr

... El sistema educativo chileno potencia esta práctica cuantificadora en los alumnos a través de las pruebas estandarizadas como el SIMCE o la PSU, siendo pruebas que no toman en cuenta los contextos educativos ni las diferencias de los sujetos. (S9)

... Cuando constatamos la presencia de una cultura de la cuantificación estamos reconociendo nuestras creencias respecto a la supuesta objetividad que es posible en los procesos evaluativos, terminando por centrar la evaluación en lo sumativo y en la certificación de logros. (S34)

Esta cuantificación, se traduce en el comportamiento del estudiantado que se moviliza en función de la obtención de calificación y el valor de cambio asociado a la evaluación; llega, incluso, a condicionar las formas de interacción en los espacios escolares.

\section{Simplificación}

Desde la cuantificación, el proceso educativo, y en consecuencia también el de evaluación, tiende a la simplificación y el reduccionismo.

En primer lugar, se constata el valor que asume la calificación como garantía y expresión del aprendizaje escolar, como los muestran los siguientes relatos:

... Aunque la Ley General de Educación declara en sus principios la igualdad y la equidad en educación, todos los niños finalmente son tratados de igual forma, se les enseña lo mismo a todos, se les evalúa igual a todos y todos terminan recibiendo un certificado con datos numéricos que nada dicen del verdadero proceso, en términos de aprendizajes efectivos. (S27)

... Todo se basa en resultados: Tasa de aprobación y reprobación, promedios semestrales y anuales, niveles de desempeño, porcentaje de cobertura curricular, pero de calidad educativa, nada o bien poco. (S15)

Al centrar la evaluación en la obtención de la calificación, se termina por desatender su potencialidad formativa, con una práctica que incurre en errores de naturaleza ética y diluye la posibilidad de impactar la mejora de los aprendizajes. Según los sujetos participantes:

... Es gracias a esta forma de ver la evaluación, que los docentes caen en errores -casi antiéticos-, como, por ejemplo, no considerar el contexto y las necesidades e intereses de sus estudiantes al momento de elaborar sus evaluaciones. No se detienen a reflexionar sobre las reales causas de la reprobación de un estudiante. Se niega el derecho de los estudiantes a ser valorados como seres particulares que requieren de atención y procesos de enseñanza que se ajusten a sus requerimientos. (S18) 
http://doi.org/10.15359/ree.26-1.2

http://www.una.ac.cr/educare

educare@una.ac.cr

... La práctica de la simplificación condiciona el cambio, puesto que no da instancias para la reflexión de los procesos de enseñanza aprendizaje, ni de las formas de evaluación y sus usos. Se centra netamente en una evaluación restringida a un momento, a un tiempo definido que busca calificar, medir y categorizar el aprendizaje. (S30)

\section{Cultura de la evaluación como comprensión}

Cuando la evaluación se concibe como un dispositivo gatillador de aprendizaje, con potencialidad para incidir en las oportunidades que tiene el estudiantado de aprender y la escuela de mejorar, emergen dimensiones que terminan por configurar una cultura distinta de la evaluación, fuerte y conscientemente demandadas por los sujetos entrevistados. Entre ellas: las posibilidades de autocrítica y reflexión de la propia práctica; el debate y la deliberación para la toma de decisiones; la flexibilidad y apertura para atender los contextos y los sujetos; y la posibilidad de constituirla en una plataforma de diálogo y encuentro.

\section{Autocrítica}

Esta dimensión de la cultura evaluativa reconocida por los sujetos entrevistados alude a la necesidad de entenderla como un espacio que, de manera natural, propicia la revisión minuciosa de la propia práctica y, con ello, da posibilidades de mejoramiento.

En cuanto espacio para la reflexión en torno a la práctica, los relatos lo esbozan en los términos siguientes:

... Este proceso es importante para detenerse y mirarse, aceptar que existen errores y podemos equivocar y nos equivocamos y que debemos reciclarnos para seguir aprendiendo. La autocomplacencia nunca genera ningún cambio, soy un convencido que los cambios más significativos ocurren desde los procesos internos de reflexión de la persona. (S7)

... Considero que la autocrítica tiene mucha potencialidad para la transformación y el cambio, permitiendo así mejorar aquello ineficiente y potenciar lo eficiente de una labor educativa y su contexto. (S13)

En lo referido a la posibilidad de mejorar, las narrativas de los sujetos reconocen, en este componente de la cultura, un espacio dotado de potencialidad formativa:

... Cuando la escuela genera espacios de reflexión que conducen a la comprensión de las situaciones, la escuela crece, pues de esa manera potencia el crecimiento profesional y se generan acciones para corregir los procesos. (S9) 
http://doi.org/10.15359/ree.26-1.2

... La evaluación debe permitir a los distintos actores de la comunidad educativa preguntarse sobre la efectividad de sus prácticas: ¿Presta el establecimiento el apoyo que requieren verdaderamente los estudiantes con necesidades educativas especiales? ¿Los docentes que trabajan con niños y niñas en el aula tienen la preparación y las capacidades necesarias para el nivel y la disciplina que imparten? ¿Permiten los ambientes de aula que los estudiantes se mantengan con disposición para el aprendizaje? ¿Es efectiva la organización curricular que existe en este colegio?, etc. (S29)

Sin embargo, el relato de los sujetos entrevistados pone de relieve la existencia de un habitus institucional con dificultades para consolidar una práctica de la autocrítica en los espacios escolares:

... La autocrítica de las propias prácticas pedagógicas es un talón de Aquiles del profesorado en nuestro país. (S40)

... La autocrítica sobre los resultados obtenidos en los procesos evaluativos, permite derribar muros de mitos y creencias arraigados en el alma de los educadores, como que "A los niños les va mal en las pruebas porque los padres no los apoyan y no estudian con ellos", o "los niños vulnerables no pueden aprender más", también "si tuviéramos más recursos los niños obtendrían mejores resultados en las pruebas". (S11)

\section{Debate}

En estrecha relación con la posibilidad de autocrítica, otra dimensión altamente valorada asociada a la evaluación es el debate, el cual es entendido como espacio de deliberación frente a las problemáticas y desafíos planteados por la práctica educativa.

En cuanto a la potencialidad de una cultura de debate, emergen relatos que destacan la comprensión que se logra de los procesos formativos y organizacionales:

... Silogramos que la evaluación se convierta en un espacio de debateentrelos distintos agentes de la educación y reflexionar sobre ella, comprenderemos sus repercusiones psicológicas y sociales, de su naturaleza y efectos. Una vez dialogada y comprendida podemos mejorarla. (S18)

... Al conversar sobre los procesos, todos quienes tengan la oportunidad de interactuar y ser parte del sistema, tendrán la opción de hacerse conscientes de las condiciones y resultados de sus prácticas, surgiendo de estos debates nuevas miradas y perspectivas de mejora. (S39) 
http://doi.org/10.15359/ree.26-1.2

http://www.una.ac.cr/educare

educare@una.ac.cr

En lo referido a las exigencias que supone, los relatos señalan:

... Necesitamos debatir sobre la evaluación y lo que está pasando con el proceso de enseñanza aprendizaje. Necesitamos responder a los diferentes contextos, ya que todas las comunidades educativas no son iguales y, por lo tanto, la evaluación también ha de responder a contextos diferentes. (S8)

... Tenemos que hacer participar a toda la comunidad educativa con el ejercicio del debate. Se trata de motivar a los apoderados y formar redes para que participen activamente y se involucren en el proceso enseñanza aprendizaje, en donde tienen que decir algo sobre la evaluación que se está practicando en dicha comunidad, así también a todos los agentes educativos. (S32)

Finalmente están identificadas las implicancias y desafíos que encierra la evaluación cuando lo que se pretende es mejorar los procesos formativos:

... La práctica deliberativa propicia la reflexión en conjunto, desde la cual se puede aprender, corregir, cambiar, mejorar. Por lo tanto, si se propicia una cultura que debate, se está propiciando indirectamente las posibilidades de desarrollo de las demás dimensiones de la cultura evaluativa y educativa. (S23)

\section{Flexibilidad}

En el reconocimiento de la autocrítica y la potencialidad del debate se advierte también el papel que pueda desempeñar la flexibilidad con la cual se asumen y resuelven los procesos formativos, los que han de ser sensibles a los contextos y sujetos, con evidente apertura y conexión.

En primer lugar, se reconoce necesaria la flexibilidad en tanto posibilidad de hacer efectiva una mirada más reflexiva de los procesos formativos:

... La flexibilidad posibilita el cambio en los procesos de aprendizaje en la medida que somos conscientes y sensibles a las particularidades de como los alumnos aprenden. (S35)

... La flexibilidad y la apertura permiten estar atento a los procesos y desde ahí poder retroalimentar a mis alumnos y mi propia práctica recordando que este es un proceso multidireccional. (S25)

En segundo lugar y estrechamente vinculada a la idea de reflexión está el compromiso con la idea de cambio, según se muestra en los siguientes relatos: 
http://doi.org/10.15359/ree.26-1.2

... Considero que la flexibilidad constituye un catalizador para el cambio y la mejora, porque genera la apertura que permite introducir modificaciones profundas, por ende, para la transformación y el cambio. (S4)

... La cultura de la flexibilidad fomenta la posibilidad del cambio educativo, porque considera el proceso de enseñanza aprendizaje como un proceso de construcción del conocimiento en el cual la interacción de los saberes y el contexto en el cual se desarrollan permiten a los estudiantes y profesores mejorar las estrategias de enseñanza, y a los educandos ser partícipes de su formación educativa. (S37)

\section{Colegialidad}

Finalmente emerge como una dimensión necesaria de intencionar a partir de la forma como se afronta la evaluación, el de la colegialidad y la colaboración, la que permite entender mejor la variedad de nuevos retos que plantea un medio ambiente cambiante a la escuela, con el objeto de asegurar adaptación y capacidad de respuesta. Esta colegialidad es entendida como el soporte donde se materializa la autocrítica, el debate y la flexibilidad.

Se reconoce que el quehacer pedagógico es situado y, por tanto, se desarrolla en un contexto sociocultural que condiciona (facilita o limita) el trabajo evaluativo.

... El estudiante, el docente, así como todas las personas desarrollan su quehacer en una comunidad, en la cual se produce el proceso de educación. Esta comunidad desarrolla ciertas prácticas y hábitos, que afectan la manera en que el profesorado lleva a cabo su labor. (S1)

... Considero que la mayor potencialidad de transformación se encuentra en la promoción de una cultura de la colaboración en la que se puede incentivar el debate y el trabajo en conjunto, que fomente el diálogo, la comprensión de la práctica, y puede permitir una cultura o una práctica distinta de la evaluación. (S17)

Por tanto, la colegialidad se constituye en una oportunidad para avanzar hacia el mejoramiento de las instituciones educativas y los procesos formativos que al interior de ellas se desarrollan. Sin embargo, los sujetos entrevistados coinciden en la necesidad de condiciones institucionales para ser desarrollada:

... El desarrollo de una práctica colaborativa en los establecimientos es un aprendizaje personal e institucional, que a la vez requiere la generación de tiempos y espacios que aseguren el encuentro, el debate y el diálogo profesional. (S21)

... Aunque en la actualidad existen comunidades educativas que reconocen los beneficios del trabajo en equipo, la verdad es que, muchos de ellos no saben cómo llevarlo a la práctica. (S19) 
http://doi.org/10.15359/ree.26-1.2

http://www.una.ac.cr/educare

educare@una.ac.cr

\section{Discusión}

Los centros educativos, a partir de sus prácticas, sus agentes y sus estructuras, crean determinados habitus (Reay et al., 2001; Tarabini et al. 2015), o culturas en los que tienen lugar los procesos de enseñanza, de aprendizaje y de evaluación. Ello significa que las propias percepciones y concepciones -tanto teóricas como prácticas- del profesorado, sobre diversos aspectos de su quehacer pedagógico, están constreñidas y coaccionadas por la institución donde trabaja y las influencias que esta genera.

Uno de los ámbitos en que opera esta influencia es el de la evaluación, la cual configura una cultura o habitus en torno a sus fines y usos. Esta evaluación puede tener un sentido positivo de apoyo y acompañamiento al aprendizaje y a la mejora de la enseñanza, o un alcance, más bien, negativo de control, clasificación y comparación. Desde la perspectiva de Perrenoud (2008), dos lógicas pueden estar presentes en la evaluación del aprendizaje en la escuela: Una al servicio del aprendizaje y, la otra, al de la construcción de jerarquías.

La evaluación de los aprendizajes, analizada en sus fines, presenta, en términos generales, una función social, de selección y de clasificación con fines de certificación y rendición de cuenta; y una función pedagógica comprometida con la noción de retroalimentación del proceso de aprendizaje y enseñanza.

Ahora bien, la existencia de nuevas formas de concebir la educación exige también nuevas perspectivas de evaluación. La evaluación enfrenta el desafío de constituirse en un espacio de mejores decisiones para promover aprendizajes, lo que supone reconocerla como:

Un proceso reflexivo, sistemático y riguroso de indagación para la toma de decisiones sobre la realidad, que atiende a su contexto, considera global y cualitativamente las situaciones que la definen, considera tanto lo explícito como lo implícito y efectos secundarios y se rige por principios de utilidad, participación y ética. (Castillo Arredondo, 2002, p. 118)

En la actualidad, la institución educativa se convierte en un encuentro de culturas, una encrucijada entre el deber ser y la realidad que provoca tensiones, restricciones y contrastes a la hora de intentar construir nuevas prácticas. En un contexto de complejidad e incertidumbre, la evaluación para los sujetos participantes del estudio se configura como una práctica paradojal, en torno a la cual es preciso reconocer la existencia de brechas entre lo que podría ser (fines y alcances) y lo que termina siendo (prácticas); dilemas entre declaraciones y prácticas y, por tanto, la permanencia de un habitus en torno a la evaluación asociada a medición, cuantificación y control; ello le resta oportunidades para transformarla en espacio de debate, reflexión, colaboración, entre otras funciones. 
http://doi.org/10.15359/ree.26-1.2

Esta cultura paradojal en torno a la evaluación se vincula al "conjunto de valores, acuerdos, tradiciones, creencias y pensamientos que una comunidad educativa asigna a la acción de evaluación" (Valenzuela González et al., 2011, p. 45), lo que termina por explicar sus aciertos y desaciertos. Siguiendo a los mismos autores, "por lo general, el discurso destaca la importancia de la evaluación, pero las prácticas y las actitudes de las personas no siempre son congruentes con ese discurso (Valenzuela González et al., 2011, p. 61).

Este habitus de la evaluación se funda en la disyuntiva entre lo existente y lo deseable; entre el habitus institucional y el individual. Desde la perspectiva de los sujetos investigados, en las instituciones prevalece una evaluación centrada en su valor de cambio, expresada así:

... Profesor, ¿el trabajo es con nota? Esta simple, pero decidora frase es el fiel reflejo de la importancia que les asignan los estudiantes a la calificación y cuantificación del aprendizaje. El hecho de que los estudiantes establezcan la relación de hacer o dejar de hacer una actividad a partir de la premisa de llevar aparejado una nota, significa un rotundo fracaso del proceso evaluativo al interior de las escuelas. (S40)

En esa perspectiva advierten que esta práctica en torno a la evaluación termina por condicionar una determinada cultura evaluativa en el sistema educativo. Por lo mismo, y en el plano de lo "deseable" se muestran conscientes:

... La autocrítica de las propias prácticas pedagógicas es un talón de Aquiles del profesorado en nuestro país. La evaluación es un elemento del curriculum de gran importancia, por lo tanto, debemos, "volver a profesionalizar" su desarrollo, desaprendiendo la cultura cuantificadora y dando cada vez más espacios a una evaluación centrada en la manera en que el sujeto aprende. (S40)

Esimportante reconocer que esta tensión que experimenta la evaluación también involucra el plano de lo explícito y lo implícito. En lo explícito, se reconocen elementos estructurales asociados a normas, reglamentos y procesos establecidos que definen los márgenes de libertad y autonomía de las acciones evaluativas; pero, a la vez y en paralelo, los sujetos actúan en un área de conflicto y poder signada por los propios intereses y creencias en sus interacciones con lo declarado, desde la institucionalidad del sistema educativo.

La evaluación que se desarrolla en los contextos organizacionales de la escuela termina por configurar una cultura que se debate preferentemente en el (des)encuentro entre lo tradicional asociado a la idea de medición y estandarización, y lo formativo, que representa la comprensión del proceso y sus condicionantes para orientar la mejora desde la autocrítica, el debate, la flexibilidad y el encuentro entre quienes participan de los procesos formativos y de gestión. 
http://doi.org/10.15359/ree.26-1.2

http://www.una.ac.cr/educare

educare@una.ac.cr

\section{Conclusiones}

Las conclusiones generadas a partir de la investigación sintetizan ciertos rasgos comunes que dan existencia a esa cultura evaluativa predominante en el sistema educativo chileno. En primer lugar, el individualismo con que operan ambos actores (profesorado y estudiantado); en segundo lugar, la competitividad que experimenta en el aula el estudiantado, en la escuela el profesorado y en la sociedad, la propia institución; en tercer lugar, la cuantificación que lleva a centrar la tarea evaluativa preferentemente en los resultados y a desatender las dimensiones potenciales del proceso; y en cuarto lugar, de manera complementaria, la simplificación y el reduccionismo reflejado en el valor que asume la calificación como garantía y expresión del aprendizaje escolar.

La evaluación, entonces, se convierte en un escenario de tensiones no resueltas a nivel de autonomía (tensión entre las pretensiones de autonomía y el control político de la sociedad como rasgo a destacar); aislamiento (que favorece la competencia e inhibe el desarrollo de objetivos educativos comunes); ausencia de relaciones colegiales y finalmente, orientación burocrática (expresada en sensación de agobio por la saturación de tareas frente a demandas y exigencias curriculares y sociales).

Estas dimensiones que caracterizan las prácticas en torno a la evaluación la tienden a alejar/ despojar de su complejidad y, por tanto, de la posibilidad de conceptualizarla y transformarla en un proceso al servicio de la toma de decisiones de naturaleza pedagógica.

Frente a las consecuencias que estas prácticas tienen en el sentido y alcance de la evaluación, los resultados de la investigación confirman que estas no favorecen la creación de una cultura de aprendizaje; por el contrario, se presentan como una experiencia, una práctica plagada de dificultades y obstáculos. Sin embargo, esas mismas contrariedades permiten visualizar la posibilidad de aprendizaje representada en sus dimensiones deseables, expresada en autocrítica, debate, flexibilidad y colegialidad.

Finalmente, para los procesos de formación inicial y continua del profesorado, la evaluación sigue constituyendo ese talón de Aquiles de la formación y la práctica, en gran parte debido a la vigencia de una serie de factores que regulan o condicionan la actividad evaluativa; entre ellos: las prescripciones legales y la filosofía que da sentido a la manera de practicar la evaluación; las supervisiones institucionales expresadas en el accionar de los agentes y dispositivos que velan por su cumplimiento; las presiones sociales que, en la actualidad, se direccionan hacia el valor del cambio más que en el valor de uso del conocimiento que se construye en las instituciones educativa; y también, las diversas condiciones organizativas asociadas a tiempos, tradiciones institucionales, exigencias de cobertura curricular $y$, en definitiva, cultura organizativa. Sin embargo, en el ámbito de las intenciones, también se perfila la necesidad urgente de convertirla en un proceso que posibilita la función pedagógica, que haga posible la integración de la unidad enseñanza, aprendizaje y evaluación. 


\section{Declaración de Material complementario}

Este artículo tiene disponible, como material complementario:

-La versión preprint del artículo en https://doi.org/10.5281/zenodo.4891347

\section{Referencias}

Alliaud, A. (2017). Los artesanos de la enseñanza. Acerca de la formación de maestros con oficio. Paidos.

Assaél Budnik, J., Cornejo Chávez, R., González López, J., Redondo Rojo, J., Sánchez Edmonson, R. y Sobarzo Morales, M. (2011). La empresa educativa chilena. Educação \& Sociedade, 32(115), 305-322. https://doi.org/10.1590/S0101-73302011000200004

Bourdieu, P. (1998). La distinción. Criterio y bases sociales del gusto. Taurus.

Castillo Arredondo, S. (2002). Compromisos de la evaluación educativa. Pearson Educación.

Chaviano Herrera, O., Baldomir Mesa, T., Coca Meneses, O. y Gutiérrez Maydata, A. (2016). La evaluación del aprendizaje: Nuevas tendencias y retos para el profesor. EduMecentro, 8(4), 191-205. http://scielo.sld.cu/pdf/edu/v8n4/edu14416.pdf

Corbin, J. y Strauss, A. (2008) Basics of qualitative research: Techniques and procedures for developing grounded theory. SAGE. https://doi.org/10.4135/9781452230153

Esquivel, J. M. (2009) Evaluación de los aprendizajes en el aula: Una conceptualización renovada. En E. Martín y F. Martínez Rizo (Coord.), Avances y desafíos en la evaluación educativa (pp. 127-143). OEl.

Flick, U. (2007). Introducción a la investigación cualitativa. Morata.

Förster, C. E. (2017). Alfabetización evaluativa de los docentes. En C Förster (Ed.), El poder de la evaluación en el aula. Mejores decisiones para promover aprendizajes (pp. 13-42. Ediciones UC.

Hargreaves, A., Earl, L. y Ryan, J. (2000). Una educación para el cambio. Reinventar la educación de los adolescentes. Octaedro.

Pérez Gómez, A. I. (2005). La cultura escolar en la sociedad neoliberal. Morata.

Perrenoud, P. (2008). La evaluación de los alumnos. De la producción de la excelencia a la regulación de los aprendizajes. Entre dos lógicas. Colhlue.

Reay, D., David, M. y Ball, S. (2001). Making a difference?: Institutional habituses and higher education choice. Sociological Research Online, 5(4), 14-25. https://doi.org/10.5153/sro.548 
http://doi.org/10.15359/ree.26-1.2

http://www.una.ac.cr/educare

educare@una.ac.cr

Rioseco Pais, M. (2016). De la domesticación a una evaluación libertaria. Reflexiones en torno a la evaluación educativa. Virtual Ediciones.

Ruffinelli Vargas, A., Cisternas León, T. y Córdoba Calquín, C. (2017). Iniciarse en la docencia. Relatos de once experiencias. Ediciones Universidad Alberto Hurtado.

Ruiz Olabuénaga, J. (2009). Metodología de la investigación cualitativa. Universidad de Deusto.

Sánchez-Sánchez, G. I. y Jara-Amigo. X. E. (2019). Los docentes en formación y la comprensión de sus experiencias pedagógicas: La evaluación del aprendizaje. Revista Internacional de Investigación en Ciencias Sociales, 15(1), 62-82. https://doi.org/10.18004/riics.2019. junio.62-82

Santos Guerra, M. Á. (1999). Sentido y finalidad de la evaluación de la universidad. Revista Interuniversitaria de Formación del Profesorado, (34), 39-59. https://dialnet.unirioja.es/ servlet/articulo?codigo $=118004$

Tarabini, A., Curran, M., y Fontdevila, C. (2015). El habitus institucional: Una herramienta teórica y metodológica para el estudio de la cultura escolar. Revista Témpora, (18), 37-58. https:// dialnet.unirioja.es/servlet/articulo?codigo $=5427947$

Tardif, M. (2004). Los saberes del docente y su desarrollo profesional. Narcea.

Tenti Fanfani, F. (2004). Viejas y nuevas formas de autoridad docente. Revista Todavía, (7), 39-42. https://issuu.com/fundacionosde/docs/todavia-07

Valenzuela González, J. R., Ramírez Montoya, M. S. y Alfaro Rivera, J. A. (2011). Cultura de evaluación en instituciones educativas. Comprensión de indicadores, competencias y valores subyacentes. Perfiles educativos, 33(131), 42-63. http://www.scielo.org.mx/scielo. php?script=sci arttext\&pid=S0185-26982011000100004

Vera Vila, J. (2007). Las relaciones escuela y comunidad en un mundo cambiante. En M. M. Castro, G. Ferrer, M. F. Majado, J. Rodríguez, J. Vera, M. Zafra y M. Zapico (Autores), La escuela en la comunidad. La comunidad en la escuela (pp. 11-37). GRAO. 